\title{
Adaptive Denoising of Event-Related Functional Magnetic Resonance Imaging Data Using Spectral Subtraction
}

\author{
Yasser M. Kadah, Senior Member, IEEE
}

\begin{abstract}
A new adaptive signal-preserving technique for noise suppression in event-related functional magnetic resonance imaging (fMRI) data is proposed based on spectral subtraction. The proposed technique estimates a parametric model for the power spectrum of random noise from the acquired data based on the characteristics of the Rician statistical model. This model is subsequently used to estimate a noise-suppressed power spectrum for any given pixel time course by simple subtraction of power spectra. The new technique is tested using computer simulations and real data from event-related fMRI experiments. The results show the potential of the new technique in suppressing noise while preserving the other deterministic components in the signal. Moreover, we demonstrate that further analysis using principal component analysis and independent component analysis shows a significant improvement in both convergence and clarity of results when the new technique is used. Given its simple form, the new method does not change the statistical characteristics of the signal or cause correlated noise to be present in the processed signal. This suggests the value of the new technique as a useful preprocessing step for fMRI data analysis.
\end{abstract}

Index Terms-fMRI, independent component analysis, principal component analysis, Rician model, signal denoising, spectral subtraction.

\section{INTRODUCTION}

$\mathbf{F}$ UNCTIONAL magnetic resonance imaging (fMRI) provides a valuable noninvasive tool for investigating brain function [1]. It localizes brain activity during mental or physical activity by detecting the corresponding increase in average cerebral blood oxygenation or cerebral blood flow [2]. To observe these hemodynamic changes, rapid acquisition of a series of brain images is performed. The sequence of images is analyzed to detect such changes and the result is expressed in the form of a map of the activated regions in the brain [1].

Classically, most of fMRI studies were conducted using the so-called block design approach, whereby two sets of data are acquired. First, a number of frames are acquired while the subject is at rest or under some baseline condition, then another set is acquired during the stimulus [1]. This pattern is repeated for a number of cycles in order to improve the

Manuscript received January 6, 2003; revised February 22, 2004. This work was supported in part by the National Institutes of Health (NIH) under Grants RO1EB00331 and Grant RO1EB002009, in part by the Georgia Research Alliance, and in part by The Whitaker Foundation.

The author is with the Biomedical Engineering Department, Cairo University, Giza, Egypt and Georgia Tech/Emory Department of Biomedical Engineering, Atlanta, GA 30322 USA. He is also with the Biomedical Imaging Technology Center (BITC), 1639 Pierce Drive, Suite 2001, Atlanta, GA 30322 USA (e-mail: ymk@ieee.org).

Digital Object Identifier 10.1109/TBME.2004.831525 signal-to-noise ratio (SNR), which would otherwise be quite low. Recent advances in both data acquisition and analysis have improved the temporal resolution of fMRI and made it possible to observe transient hemodynamic changes with reasonable accuracy. A good example for that is a new experimental design, similar to that of evoked-response potential (ERP) protocol, called single trial or event-related fMRI (ER-fMRI). In this new design, the subject receives a short stimulus or performs a single instance task while the resultant transient response is measured [3]. ER-fMRI offers many advantages over block design that include versatility, investigation of trial-to-trial variations, and extraction of epoch-dependent information and direct adaptation of the methods used for ERP to fMRI. The main drawback of ER-fMRI is the degradation in SNR due to the transient nature of the response. As a result, such studies now include epoch averaging [3]. Nevertheless, this comes at the expense of suppressing the information about intra-subject variations related to psychophysiological function with each execution of the task. Therefore, a processing method that can be used to suppress noise in the acquired data would be very useful to reduce the experiment duration and preserve the information within the acquired data [3], [4].

Several methods of data analysis have been used to process the fMRI raw data. The ultimate goal of such analysis is to try to separate signal components due to true activation, physiological fluctuations and random noise. The latter two components are considered as nuisance and must be removed for correct results [7]. Several methods have been proposed to suppress physiological noise including the use of harmonic model [5] and noise subspace characterization [6]. Others attempted to use different strategies to suppress the effect of random noise in the analysis using finite impulse response (FIR) filter modeling [8], smart spatial averaging [9], inter-epoch averaging [3], and Wiener filtering [4]. These techniques suffer from at least one of the following limitations: the need for extended data acquisition for inter-epoch averaging that might not be practical, assumption of limited epoch-to-epoch variability, dependence on assumptions about the signal characteristics to build the denoising filter, and the inability to use conventional statistical detection approaches because of correlated noise among spatial and/or temporal points. Therefore, a denoising strategy that does not have the above limitations would be rather useful in the clinical practice.

Among the most powerful techniques that can be used to separate signal components are those based on blind source separation such as principal component analysis (PCA) and independent component analysis (ICA). These techniques decompose 
the signal sources using either the second order statistics (as in PCA) or higher order statistics such as the kurtosis (as in ICA) to account for the non-Gaussian nature of the sources [12], [13]. According to the assumptions of both techniques, the number of independent signal components must be less than or equal to the number of signals to be analyzed [13]. Otherwise, the separation of components yields incorrect results or even may not converge at all as in ICA. Unfortunately, this condition is not satisfied in fMRI data sets. Given the general assumption of uncorrelated noise, the number of components of random noise alone is equal to the number of signals. The total number of components has to add the number of components due to physiological fluctuations as well as the activation components. As a result, the use of PCA and ICA based techniques may not be successful in practice unless the noise signals are sufficiently weak. This may account for the limited use of such techniques in low SNR applications [13]-[17]. Therefore, a technique that suppresses random noise or removes some of its components would be rather useful for making the use of PCA and ICA more robust for clinical practice.

In this paper, we study the problem of reducing the random noise while preserving the other deterministic components in fMRI signals. A new adaptive technique is proposed based on spectral subtraction. This technique relies on the observation that accurate information about the random noise model parameters can be easily obtained adaptively from ER-fMRI data sets. Given this information, the new technique uses the uncorrelatedness of the random noise and the deterministic components of the signal to separate the two in the original power spectrum. The theoretical analysis of the new technique and the implementation details are presented. The new technique is tested using computer simulations as well as real data and the performance is analyzed. Finally, the value of the proposed method as a preprocessing stage for blind source separation techniques such as PCA and ICA techniques is demonstrated.

\section{THEORY}

Generally speaking, the fMRI temporal signal can be modeled as the summation of the true activation signal, a physiological baseline fluctuation component, and a random noise component. The physiological baseline fluctuation component can be considered as a deterministic yet unknown signal. Therefore, we will consider a model that is composed of the sum of one deterministic component $d(t)$ incorporating both the true signal and the physiological noise and an uncorrelated stochastic component $n(t)$. That is

$$
s(t)=d(t)+n(t) .
$$

Since these two component are assumed independent, the corresponding power spectra are related by

$$
P_{s s}(\omega)=P_{d d}(\omega)+P_{n n}(\omega)
$$

where cross terms vanish because the two components are assumed uncorrelated. Hence, an estimate of the power spectrum of the deterministic component takes the form [10]

$$
P_{d d}(\omega)=P_{s s}(\omega)-P_{n n}(\omega) .
$$

That is, the signal power spectrum is obtained by spectral subtraction of the noisy signal and noise power spectra. In order to compute the deterministic signal component from its power

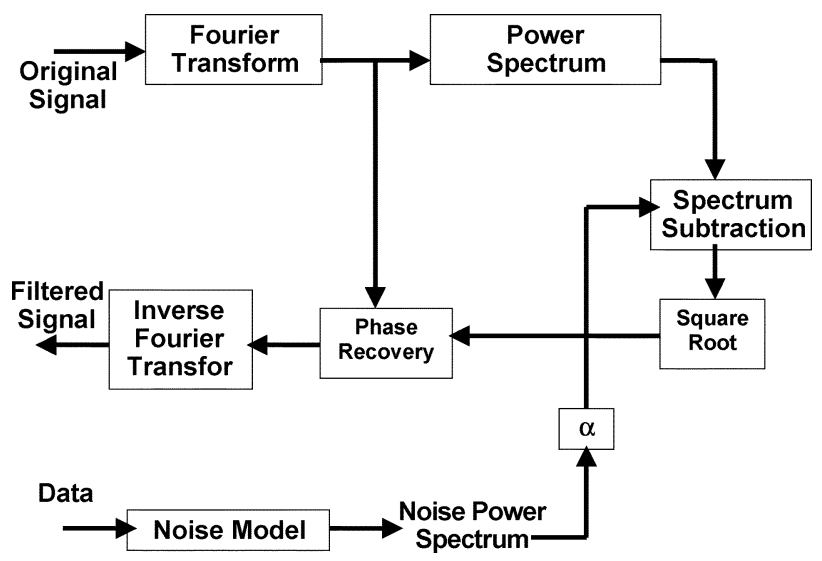

Fig. 1. Block diagram of the proposed technique.

spectrum, the magnitude of the Fourier transform can be obtained as the square root of the power spectrum. The problem now becomes that of reconstructing the signal using magnitude only information about its Fourier transform. Several techniques can be used to do that. The one used for this paper relies on an estimate obtained from the phase of the Fourier transform of the original signal $S(\omega)$. Hence, the Fourier transform of the processed signal $S_{d}(\omega)$ can be expressed as

$$
S_{d}(\omega)=\sqrt{P_{d d}(\omega)} \cdot e^{j \operatorname{Phase}(S(\omega))} .
$$

The enhanced deterministic signal $s_{d}(t)$ is then computed as the real part of the inverse Fourier transformation of this expression. It should be noted that this method is related to the optimum Wiener filter. A detailed discussion of that is presented in the Appendix. A block diagram for the proposed strategy is shown in Fig. 1.

\section{FMRI NOISE POWER SPECTRUM MODEL}

In order to implement the above denoising strategy, the noise power spectrum has to be estimated. Our strategy is to use the background pixels in fMRI images to estimate this model given that they contain only noise. Nevertheless, the noise model in fMRI data sets belongs to the Rician distribution. A unique feature about this distribution is that its characteristics depend on its mean value ranging from the Rayleigh distribution when the mean is zero to near Gaussian distribution when the mean is large enough. In the following, we describe the characteristics of this model and show that it is still possible to use the noise characteristics in the background to derive useful information about the noise model inside the brain.

\section{A. Rician Model Characteristics}

The raw MRI data consists of complex valued samples with real and imaginary parts that can each be modeled as the summation of deterministic and a Gaussian random noise components. For fMRI, which relies on image magnitude, the noise within each pixel belongs to the Rician distribution [23]. This distribution form depends in a complex manner on the value of the means of the real and imaginary part distributions [19], [23], which are unknown (in fact their values represent the solution for the denoising problem). The power spectrum of this model can be computed as the Fourier transform of the autocorrelation function of the data $A(\tau)$. For the case when $\tau=0$, the 

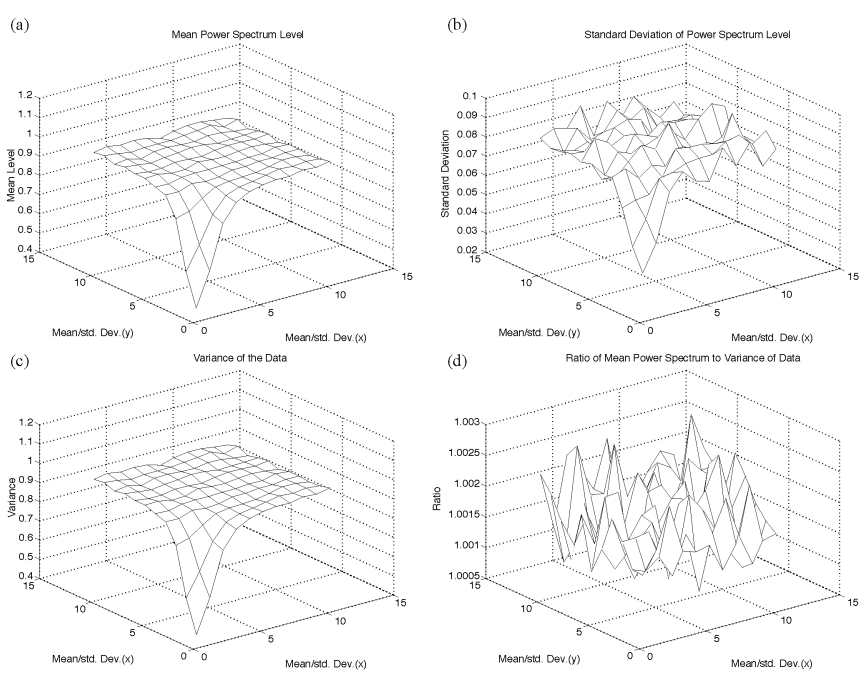

Fig. 2. The simulation of power spectrum flat level for Rician distributions with different components means. The $\mathrm{x}$ and $\mathrm{y}$ axes represent the ratio of the mean to the standard deviation of the noise in one of the quadrature components. (a) The mean power spectrum level (b) the standard deviation of the mean level (c) the variance estimated directly from the data (d) the ratio of the mean power spectrum level and the variance of the data.

autocorrelation function $A(0)$ is the just the second moment of the data (which is a function of the variance of Gaussian noise components and the mean values of the components as defined by the Rician distribution). On the other hand, for all other autocorrelation values where $\tau \neq 0$, given the independence and identical distribution of samples, all values of $\tau$ other than 0 provide identical mathematical expressions and consequently should be equal in value. Hence, the autocorrelation function will take the form of a flat distribution with a different value only at $\tau=0$. Consequently, the power spectrum takes the form of a delta function at the dc value corresponding to the level of the flat part, in addition to a flat function with a level corresponding to the difference between the second moment and the flat part in the autocorrelation function. So, since the de value is not important for our filter, the task of estimating the noise power spectrum is now that of estimating a single value of this flat level.

\section{B. Practical Noise Model Estimation}

In order to estimate the flat level of the Rician noise power spectrum, we observe that the numerical evaluations of the Rician distribution in the literature exhibit special characteristics when the means of the components are much larger than their standard deviations [19]. A numerical simulation of the fMRI signal generation was performed whereby two quadrature components with variable means and similar standard deviations of unity were used and the resultant power spectrum was computed for all values of the mean (or equivalently in our case the mean over standard deviation). The time course lengths used were $64,128,256,512$, and 1024 points consecutively and the results were averaged over 128 time courses in each. The power spectrum is estimated using the periodogram method as the square of the absolute value of the discrete Fourier transform of the time course. The orthogonal discrete Fourier transformation matrix [20] was used and the results of this simulation for the case of 512-point time courses are shown in Fig. 2. The mean value of the power spectrum flat level in Fig. 2(a) and

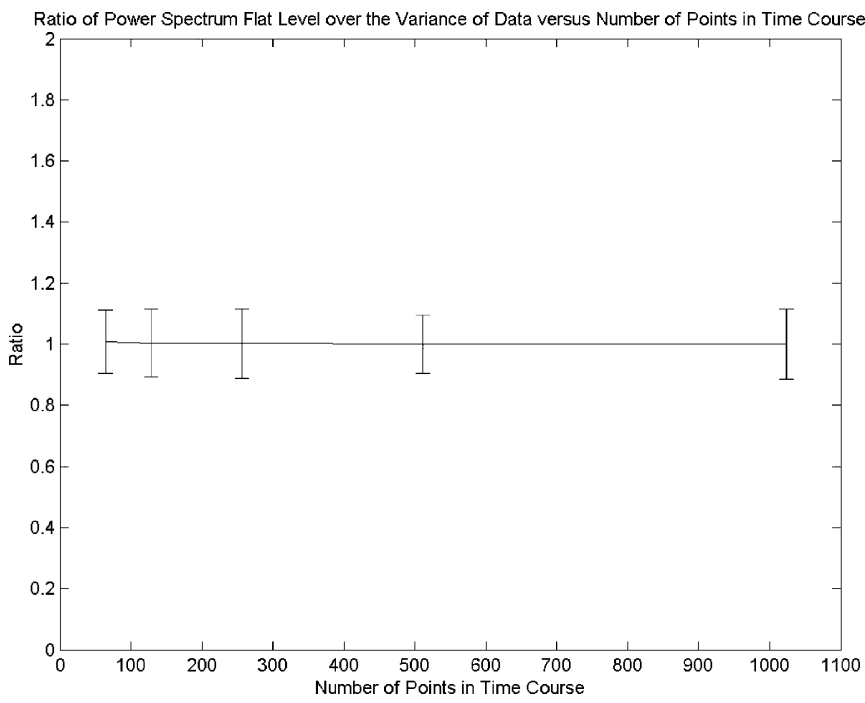

Fig. 3. A plot of the factor of the mean power spectrum flat level divided by the variance calculated from the data versus the number of points in the time course. The curve looks very close to a linear curve with a slope of zero.

its standard deviation in Fig. 2(b) are plotted versus the ratios of mean value of each quadrature component to its standard deviation. It can be noticed that the values of the mean above approximately five times the standard deviation result in nearly similar levels for the flat region. Moreover, if we look at the plot of the calculated variance of the data in Fig. 2(c) and the division of the mean power spectrum level over this variance in Fig. 2(d), we observe that the result is a flat curve over all values of the component means. Also, the results relating the mean of the flat part in each to the number of points is shown in Fig. 3. The linear regression of the data provided a formula for the noise power spectrum model as a linear curve with a slope of $-5.7250 \mathrm{e}-6$ and an intercept of 1.0053 . The $95 \%$ confidence intervals for this regression are $[-0.1518 \mathrm{e}-5,3.7279 \mathrm{e}-6]$ for the slope and [1.0003, 1.0103] for the intercept. Hence, all is needed to compute the Rician noise power spectrum is to compute the variance of an ensemble of the data that need not have the same mean as the time course of interest. This conclusion substantially simplifies the implementation of the proposed denoising method since it shows that the background pixel time courses can still be used to estimate a model that would be applicable to brain time courses.

\section{METHODS}

\section{A. Adaptive Parameter Estimation of the Noise Model}

According to the above derivation, we need to compute the variance of the data in order to obtain the noise power spectrum model using the empirical formula. The simplest way to do that is to estimate the variance of background areas within the available data set. In order to avoid bias errors from baseline variations in calculating the noise variance, the variance is computed from the selected background pixels within each image in the sequence separately. Then, the average of the obtained variance values from all images is taken to be the noise model variance estimate. This simple procedure allows baseline variations among the images in the sequence to be eliminated thus providing a more accurate estimate for the noise model. Fig. 4 shows the calculated variance estimates along the time direction 
(a)

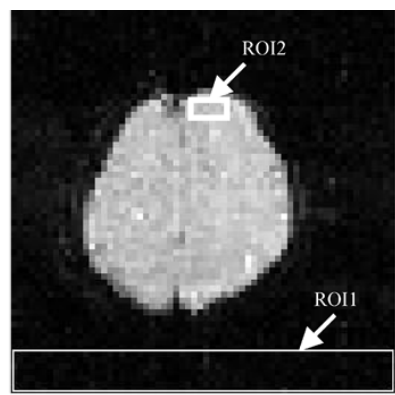

(b)

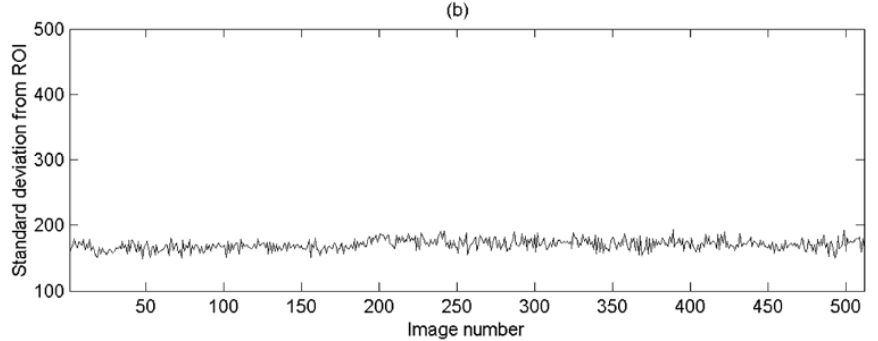

(c)

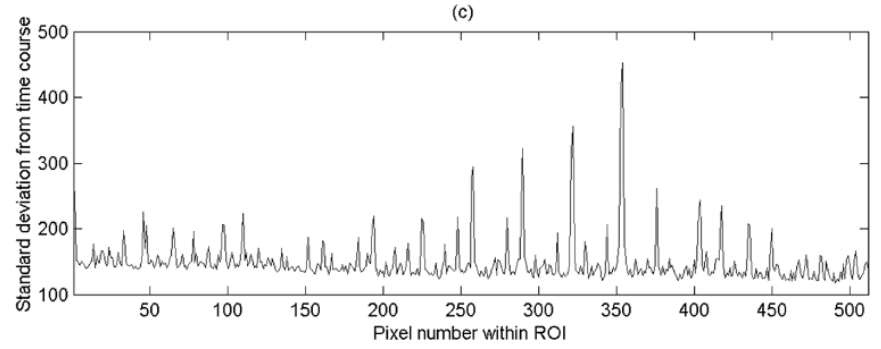

Fig. 4. Estimation of parametric noise model from data. (a) Designated region of interest (ROI) for noise model calculation (ROI1) and brain ROI used to derive the time courses for processing (ROI2). (b) Calculated noise standard deviation based on ROI versus image number. (c) Calculated noise standard deviation from time courses of pixels within ROI versus pixel number. Large fluctuations are observed in (c).

and between different time courses to demonstrate the stability of the proposed method. As can be seen, the variance computation from temporal time courses in Fig. 4(c) shows undesirable fluctuations as a result of the presence of baseline variations. On the other hand, the calculation of variance from spatial points within the same image illustrated in Fig. 4(b) appears to be more robust since the baseline variations do affect pixels within the same image.

\section{B. Signal Power Spectrum Estimation}

Since the proposed technique is applied to a single time course at a time, the periodogram estimate of signal power spectrum is expected to have a rather large variance [20]. As a result, the subtraction of power spectra in (3) may contain negative values in practical implementations. This causes a problem in trying to compute the square root to recover the processed signal. The approach used in our implementation to overcome this problem relies on replacing all negative values in the subtraction results by zero. This approach is justified because all values lower than the estimated power spectrum are more likely to be noise components within the variance limits of the periodogram estimate.

\section{Control Over Level of Denoising}

Given the nature of the original signal, we observe that the variance in the power spectrum estimate may only result from the random component. Since the expected value of the noise variation is known from the derived model and given the statistical characteristics of the periodogram estimate, we can express the noise at each of the power spectrum frequency bins as a Gaussian random variable with mean and variance both equal to the noise model [10], [20]. As a result, the subtraction in (3) would effectively remove only a part of the noise power spectrum. In other words, the upper half of the Gaussian distribution would still remain in the processed signal.

To solve this problem, a slight modification to the technique is added to allow direct control over the extent of noise removed. The modified equation takes the form

$$
P_{d d}(\omega)=P_{s s}(\omega)-\alpha \cdot P_{n n}(\omega) .
$$

Here, the factor $\alpha$ is added to control the confidence of noise removal. This problem can be expressed in the form of a statistical $z$ test where $\alpha$ controls the p-value of the test. That is, the larger the value of $\alpha$, the less the probability that the output power spectrum contains a noise component. On the other hand, increasing this value would increase the likelihood that some parts of the signal may also be removed. Therefore, the selection of the value of $\alpha$ is useful to fine-tune the results of the new technique. Several optimization criteria can be used to select the value of this parameter. An example of these is the use of entropy based objective function optimized over the autocorrelation function of the difference between the original and processed signals for different $\alpha$ values. This favors the values of $\alpha$ that give an autocorrelation function with narrow extent around zero and of minimal side peaks. This tends to preserve the components of the true signal, which give rise to periodic peaks in the autocorrelation function. Another approach is to optimize the kurtosis of the difference as close as possible to zero to make sure that the removed signal is only the Gaussian random noise component. In this paper, we used a fixed value of this parameter that is equal to 1 to make it easier to compare the results and assess the improvement after using this technique as a preprocessing stage and the optimization of this parameter will be left for further investigation.

\section{Practical Implementation Steps}

The following are the steps needed to implement the spectral subtraction denoising procedure in practice.

Step 1) Select a spatial area within the background part of the image outside the brain away from the Nyquist ghosts and obtain the time courses corresponding to each point within this area.

Step 2) Compute the variance of the intensity values of this area at each time point. Averaging the estimate from all time points to obtain the noise power spectrum level.

Step 3) For each point in the image, compute the Fourier transform of its time course and save the phase and magnitude parts of the result separately.

Step 4) Compute the original power spectrum of this time course using the periodogram method as the square of the magnitude of the Fourier transform in Step 3.

Step 5) Compute the denoised power spectrum by subtracting the noise power spectrum from Step 2. Observe any scaling factors that are introduced by the Fourier transform definition before performing 
the subtraction in addition to the factor $\alpha$ in (5) (use a default value of $\alpha=1$ ).

Step 6) Compute the denoised signal Fourier transform as the square root of the denoised power spectrum from Step 5 multiplied by the phase retained in Step 3.

Step 7) Compute the denoised time course as the real part of the result of Step 6.

\section{E. Applications}

The new method can be used as a preprocessing step for different fMRI analysis methods including parametric approaches (e.g., cross-correlation analysis) as well as nonpareametric techniques (e.g., PCA and ICA). For conventional methods, spectral subtraction is applied before other post-processing steps in order to enhance the detection of activation signals for low SNR acquisition methods such as EPI. On the other hand, when using PCA/ICA, the new technique is applied to process all pixel time courses in the acquired data set independently and then the processed data set is used for subsequent PCA/ICA. The results are analyzed to assess the performance of the new technique in enhancing the results of PCA and ICA and stabilizing the convergence characteristics of the ICA especially when a small region is analyzed.

\section{RESULTS}

The proposed technique was verified using computer simulations as well as actual data from a human volunteer. The computer simulations were performed in a similar fashion to [4] whereby a computer generated ER-fMRI activation signal was added to an actual baseline data set. The baseline data were collected on a healthy human volunteer using an EPI sequence $(\mathrm{TE} / \mathrm{TR}=25 / 500 \mathrm{~ms}$, Matrix $=64 \times 64$, field of view $($ FOV $)=20 \mathrm{~cm} \times 20 \mathrm{~cm}$, slice thickness $=5 \mathrm{~mm}, 640 \mathrm{im}$ ages) on a Siemens Magnetom Vision 1.5 T clinical scanner. The number of epochs was 8 and the length of each epoch was 64 . The generated activation was designed to include inter-epoch variations in both the magnitude and width of the activation signal in order to test the performance of the new technique in preserving such variations. The overall standard deviation of the generated activation was varied to test the performance under different values for SNR in a similar fashion to [4].

The actual data were obtained from an ER-fMRI study performed on a normal human volunteer using a Siemens $1.5 \mathrm{~T}$ Magnetom Vision clinical scanner [4]. In this study, an oblique slice through the motor and the visual cortices was imaged using a T2 $2^{*}$-weighted EPI sequence $(\mathrm{TE} / \mathrm{TR}=60 / 300 \mathrm{~ms}$, flip angle $=55^{\circ}$, Matrix $=64 \times 64, \mathrm{FOV}=22 \mathrm{~cm} \times 22 \mathrm{~cm}$, slice theckness $=5 \mathrm{~mm}$ ). The subject performed rapid finger movement cued by flashing LED goggles. The study consists of 32 epochs with 64 images per epoch. Temporal data from only 4 or 8 epochs of pixels in both the motor and visual cortices were processed using the new method and compared to the case when the remainder of the acquired epochs are used for averaging. The PCA and ICA techniques were applied to decompose the signal into its basic components. Both techniques were used to process time course signals before and after the new technique is applied on pixels within a window selected by
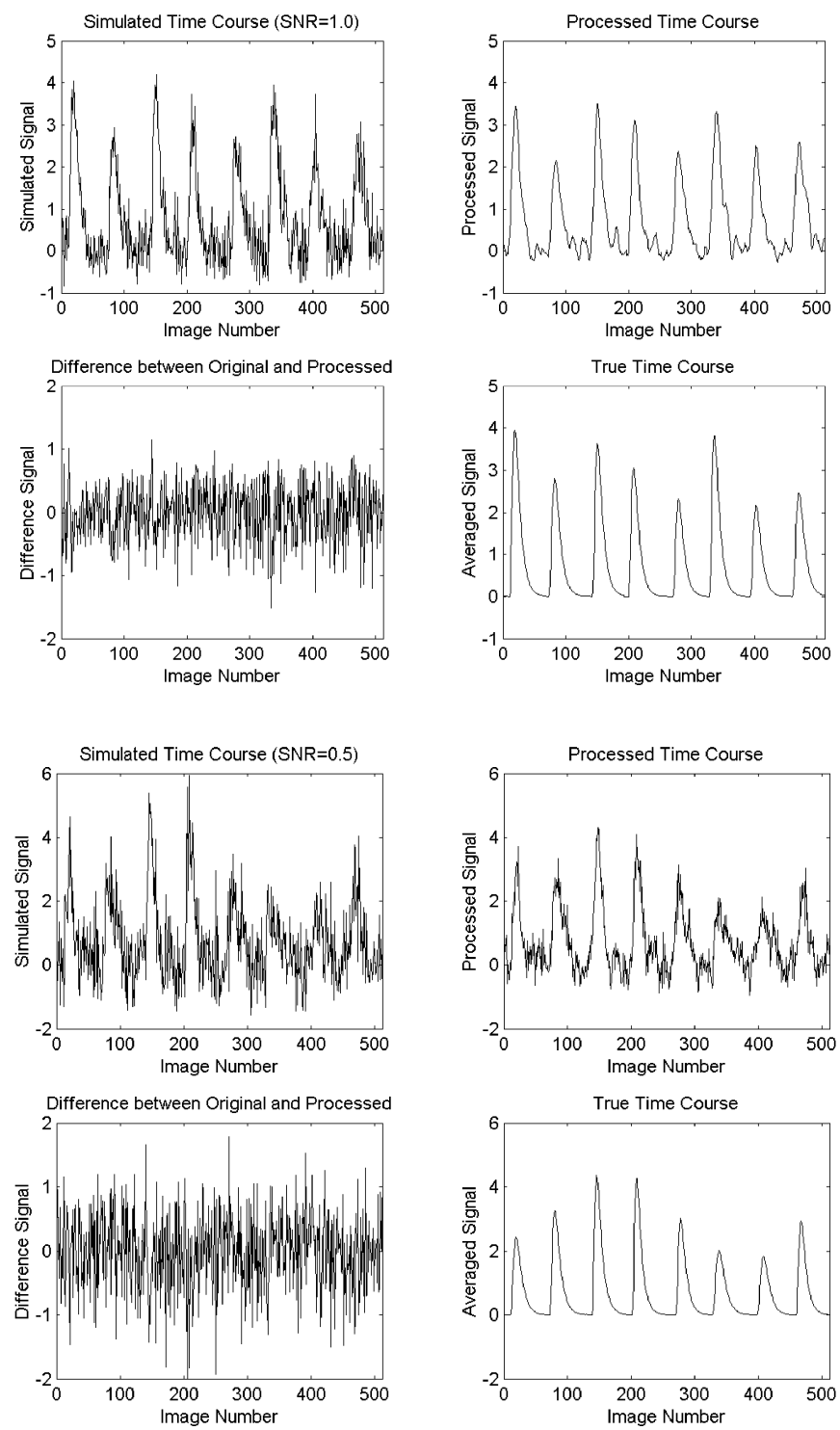

Fig. 5. Results from simulations with SNR values of 0.5 and 1.0.

the user. Moreover, the difference signals were also analyzed from the same window.

The results of applying the new technique to process computer simulated fMRI data are shown in Fig. 5 for SNR values of 1.0 and 0.5 . As can be observed, the noise in the original data was suppressed significantly in the output and the difference signal appears free of signal components. In order to compare the new technique to previous methods in a controlled study, the simulations were repeated 100 times and the resultant signal from each simulation was processed using spectral subtraction, wavelet-domain Wiener filtering and inter-epoch averaging of 8 epochs. The root-mean-square (rms) error was computed between the true simulated signal and the four signals including the original and the signals processed with the above three methods. The simulation was repeated for SNR values of $0.25,0.5,1,2$, and 4, and the results were plotted in Fig. 6. The results indicate that the performance of the new method is comparable to that of the Wiener filter approach and seems to provide better results that inter-epoch averaging for low SNR. Notice that the Wiener filter used here was designed given the 


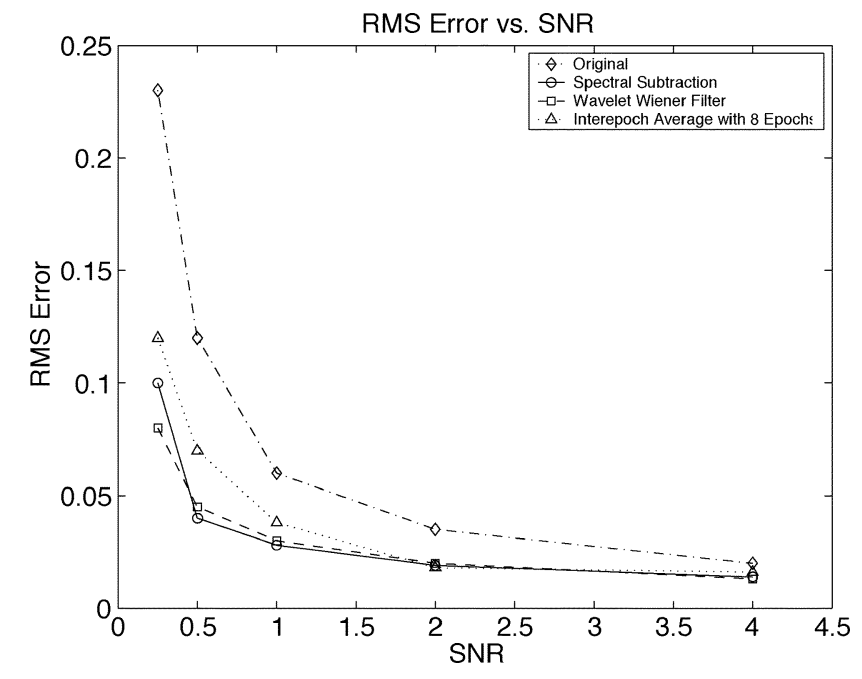

Fig. 6. Comparison between the rms error in the original signal, the signal processed using spectral subtraction, the signal processed with wavelet-based Wiener filter, and the inter-epoch average with 8 epochs.

knowledge about the true signal, which is generally not the case in practical fMRI data processing. Given that the spectral subtraction denoising procedure did not depend on any assumption about the nature of the present signal, it is expected to maintain its performance under such practical conditions.

In Figs. 7 and 8, the proposed technique is used to process real fMRI data from two activated pixels with time course lengths of 512 and 256 points (i.e., correspond to 8 or 4 epochs). The results look dramatically improved compared to the original. In fact, they appear even less noisy than the result from inter-epoch averaging while maintaining the signal structure unaltered. We also notice that the baseline variations also remained unaltered. Again, the difference signals appear to have no signal components. Also, the filtered signal looks very similar to that obtained using inter-epoch averaging with the exception of losing the baseline and inter-epoch variations as a result of this averaging.

In Fig. 9, the results of applying spectral subtraction to process a sample time course for a nonactivated pixel in the same data set are shown. As can be observed, the processing removed a significant amount of the random variations while keeping the baseline variations intact. This emphasizes the fact that this processing technique reduces only random noise while preserving the deterministic parts of the signal-including physiological noise and baseline variations. To illustrate this even further, the power spectra of the signal before and after processing with spectral subtraction are shown in Fig. 10. The signal in this example is the same as the bottom half of Fig. 7 after removing the dc shift for better visualization. The bottom row shows the same power spectra as the top row using a different scale to illustrate the effect of spectral subtraction. As can be seen, the denoised spectrum is just a downshifted version of the original with the negative parts clipped. Notice that the peaks corresponding to the fundamental frequency and harmonics of the activation signal as well as those due to baseline variations are still present in the processed power spectrum. This shows the difference between this approach and low-pass filtering, which would generally result in losing the higher frequency peaks and causing signal distortion.
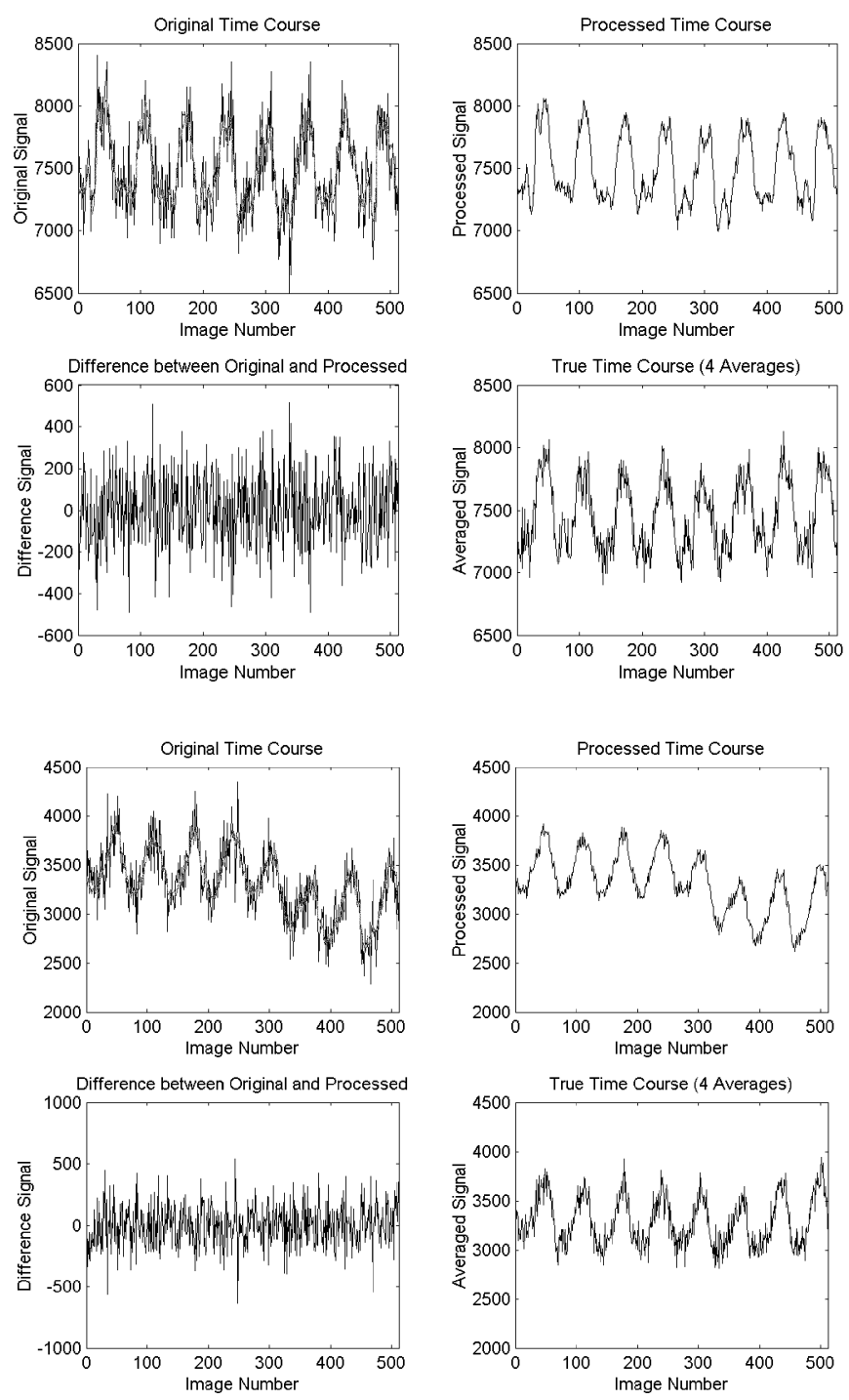

Fig. 7. Results from actual data for two pixel time courses containing neuronal activation (length $=512$ points).

Fig. 11 shows the results from denoising the same time course using different values for $\alpha$ including the value of 1 used to generate all the results in this paper. As can be seen, increasing the value of this parameter results in losing some of the weak harmonics in the signal. This is particularly apparent in the lower two figures as compared to the upper two.

To illustrate the possible practical applications of the new method, two examples are presented here. The first is the preprocessing of fMRI data sets before parametric activation detection and the second is preprocessing for nonparametric techniques. In Fig. 12, Comparison of fMRI activation detection results without (a) and with (b) spectral subtraction preprocessing using the same parameters including the same statistical parameter map threshold. The paradigm used consisted of the following parts in sequence: left motor, language, right motor, and language, with 10 images acquired in each. This paradigm was repeated four times. The experiment used a gradient echo EPI sequence with the following imaging parameters: TR/TE $=$ $3000 / 30 \mathrm{~ms}$, Matrix: $64 \times 64$, FOV $=220 \mathrm{~mm}, 32$ slices with slice thickness of $3 \mathrm{~mm}$ and distance factor of $0 \%$. The data were collected on a Siemens Trio $3 \mathrm{~T}$ scanner. The analysis 

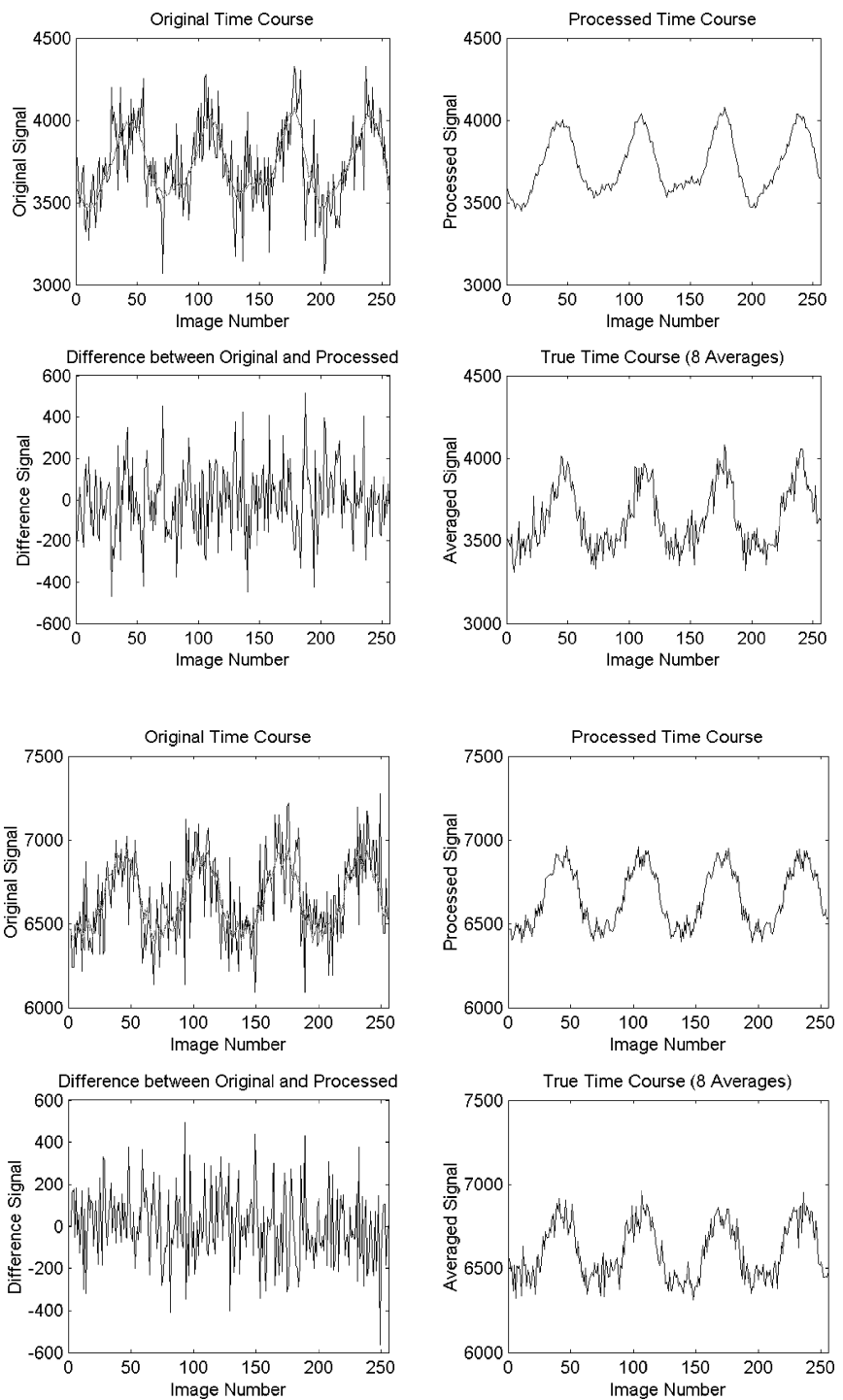

Fig. 8. Results from actual data for two pixel time courses containing neuronal activation (length $=256$ points).

was done using Brain Voyager software (Brain Innovation B.V., Maastricht, The Netherlands). The threshold was set at correlation $r>.4$ for both maps and the maps were overlaid on the original data. Both sets of data were motion corrected, slice timing corrected, and then the time courses of a spatial-temporal Gaussian kernel of size $5 \mathrm{~mm} \times 4 \mathrm{~s}$ is used to derive the maps. The only difference between the two maps was the application of spectral subtraction as a preprocessing step for the second map before all other steps. The results indicate that the sensitivity of the conventional analysis after denoising is clearly higher even though the energy of the denoised time courses is generally lower. Observe also that the activations shown in the second map seem to be localized and correspond to the brain areas involved in this paradigm. We believe that this sensitivity is due to two compounded effects of reducing the random noise contents in addition to enhancing the performance of other preprocessing steps such as motion correction, which work better on denoised data. This is an additional advantage of this method since it does not require this registration step to be performed
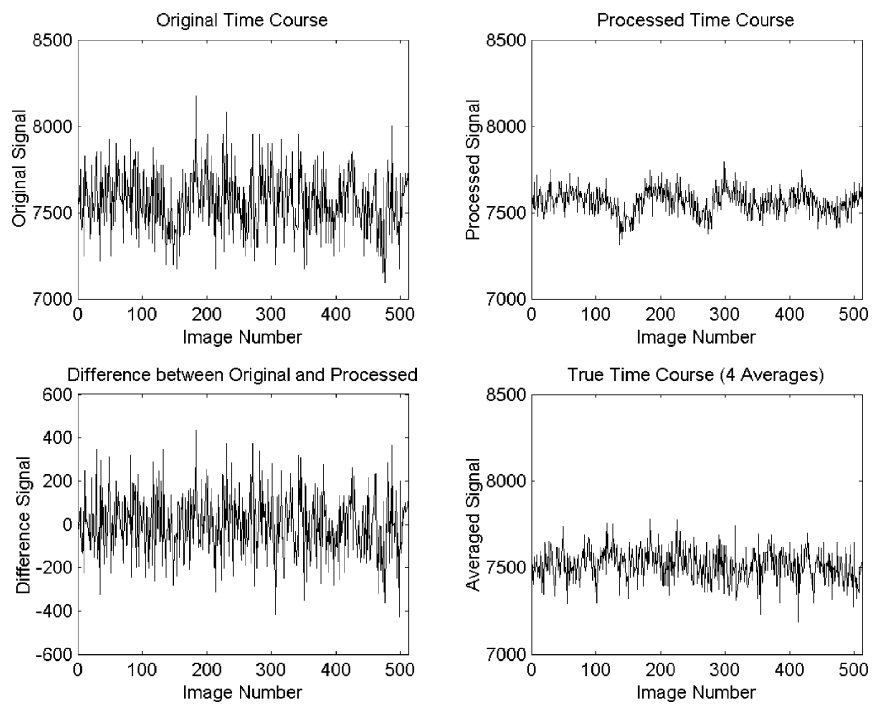

Fig. 9. Results from actual data for a pixel time course containing no activation signal (length $=512$ points).
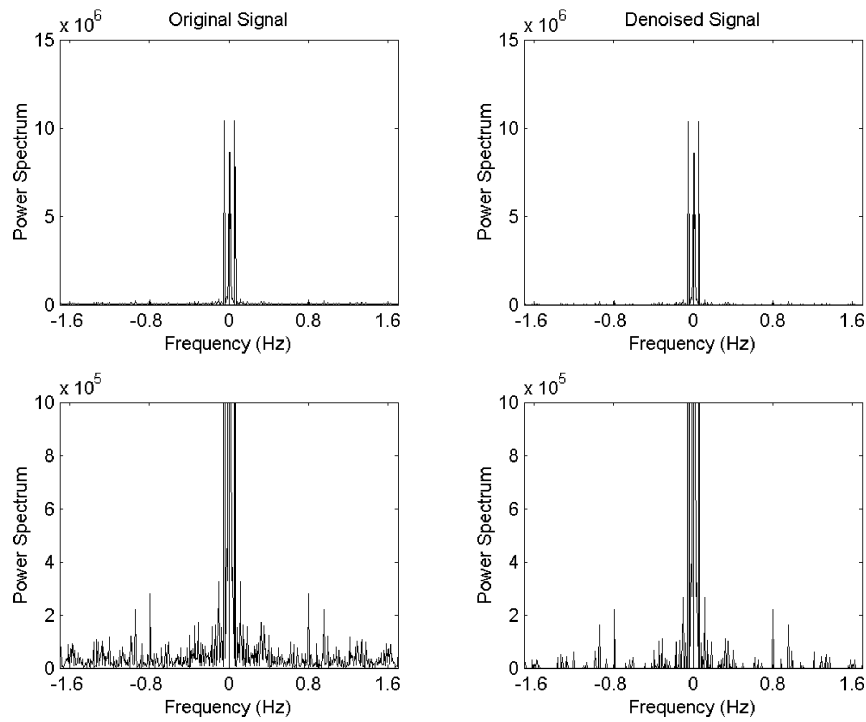

Fig. 10. Illustration of the power spectra of the original signal and the denoised signal using spectral subtraction for the time course shown in the bottom half of Fig. 7. The bottom row shows the same power spectra in the top row using a different time vertical scale.

prior to denoising as all other techniques given that the noise model is same and that only the activation signal is affected.

In Figs. 13 and 14, the results of applying the PCA and ICA techniques before and after processing with the new technique are presented. The PCA/ICA techniques were performed on the time courses of local regions using a Matlab (Math Works, Inc.) program based on the FASTICA algorithm [18]. The application of the proposed method is done in the case when the real time course data without loss of generality to complex time courses [17]. The PCA and ICA were applied to the time courses of pixels within a local region of size $4 \times 4$ around an activated pixel in the acquired data set. The figures show the first four components of the PCA and four selected components from the ICA results (notice that ICA involves a whitening step that removes any preference of one independent component over the 


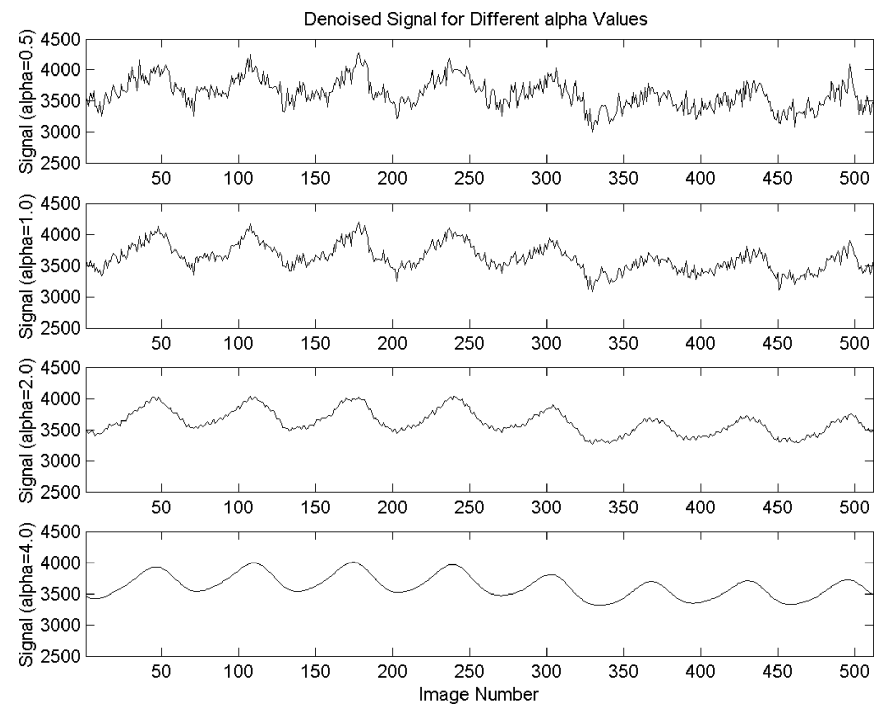

Fig. 11. Application of the new technique for different values of $\alpha$. As $\alpha$ becomes larger, some of the weak harmonics of the actual signal are removed.

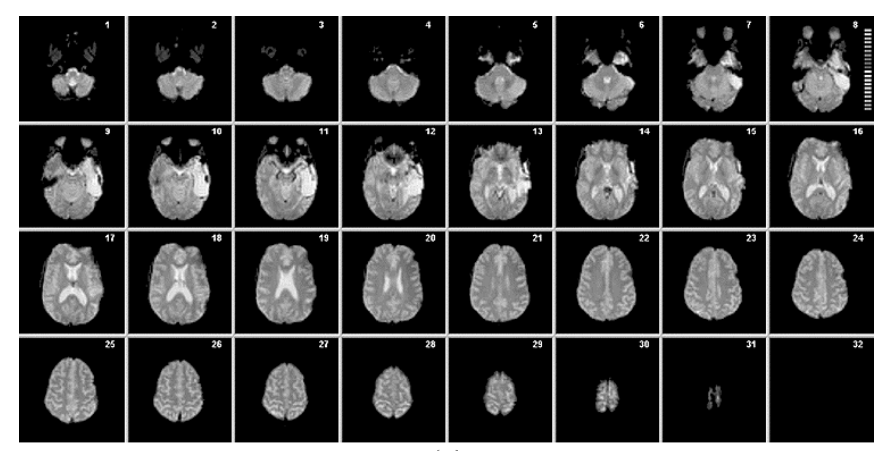

(a)

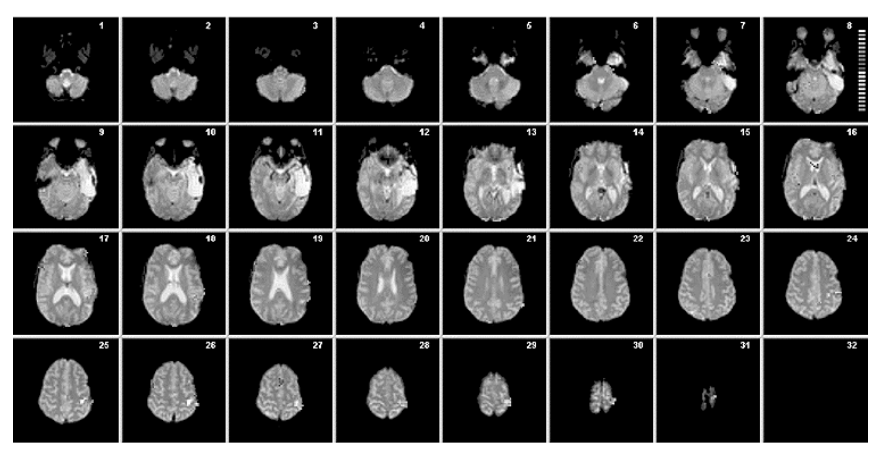

(b)

Fig. 12. Comparison of fMRI activation detection results without (a) and with (b) spectral subtraction denoising using the same parameters including the same statistical parameter map threshold.

other unlike PCA). The results appear very noisy before applying the new technique in both methods. The results after it was used appear significantly clearer. We notice that the baseline variation component now appears in the ICA results, which was not present in the analysis before denoising. The results of applying PCA on the difference signal are shown in Fig. 15. As can be observed, no periodic components appear on the first four principal components indicating the random nature of the removed signal. Moreover, severe instability (i.e., no convergence of the ICA iteration) was observed when using the ICA iteration

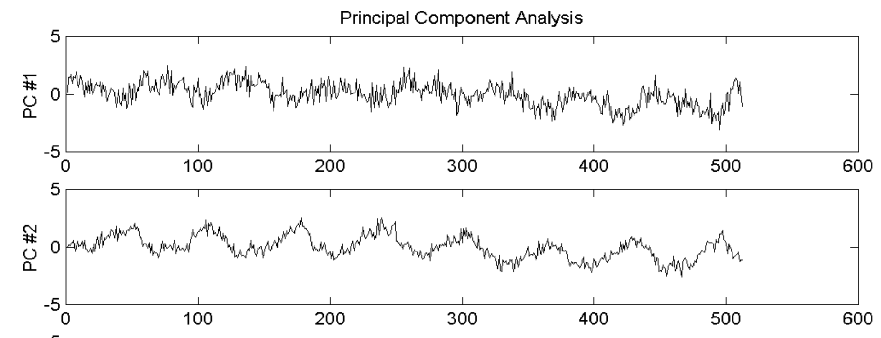

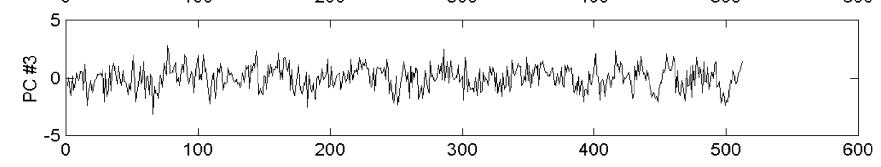

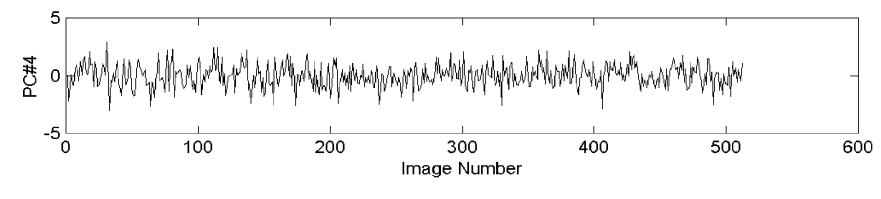

Independent Component Analysis

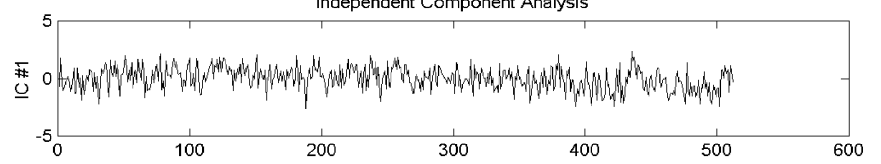

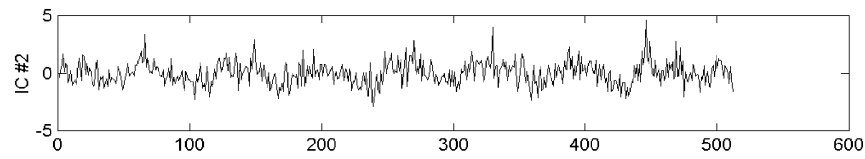

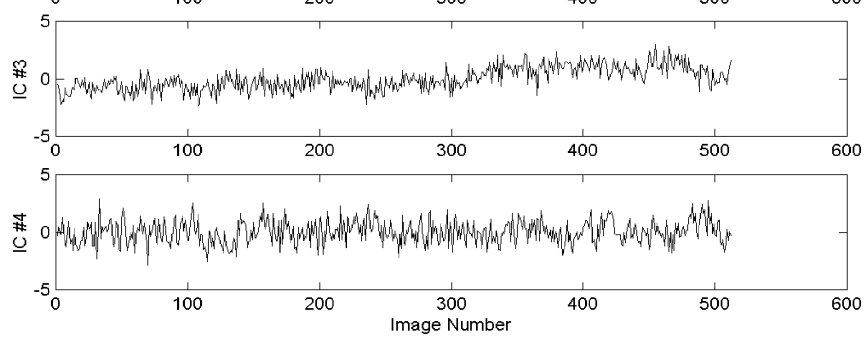

Fig. 13. PCA/ICA results before processing with the proposed method.

on this difference data. This is expected given the known limitation of ICA when the components are random signals or when the SNR is very low.

\section{DISCUSSION}

The results of using the new technique suggest that it suppresses random noise while preserving deterministic signal components. Because it relies on subtracting the noise component (if we ignore the small nonlinear effect of clipping the negative values in the power spectrum), it does not generally affect independence of data points within the time course. This allows the technique to be virtually transparent to conventional statistical analysis methods, which assume statistical independence of samples. This is important for further analysis of the fMRI data (c.f., activation detection [4] and motion correction of fMRI time series [21]). This means that no constraints are imposed on the data analysis when the new method is used as a preprocessing step.

The technique also has a very small computational complexity. Assuming a data set of $\mathrm{L} \mathrm{NxN}$ images, the process of noise model estimation requires a computational complexity of nearly $\mathrm{O}(\mathrm{L} \cdot \mathrm{N})$. The actual denoising of a single time course requires $\boldsymbol{O}(\mathrm{L} \cdot \log (\mathrm{L}))$ computations. This means that the whole data set can be denoised using $\boldsymbol{O}\left(\mathrm{N}^{2} \cdot \mathrm{L} \cdot \log (\mathrm{L})\right)$ 

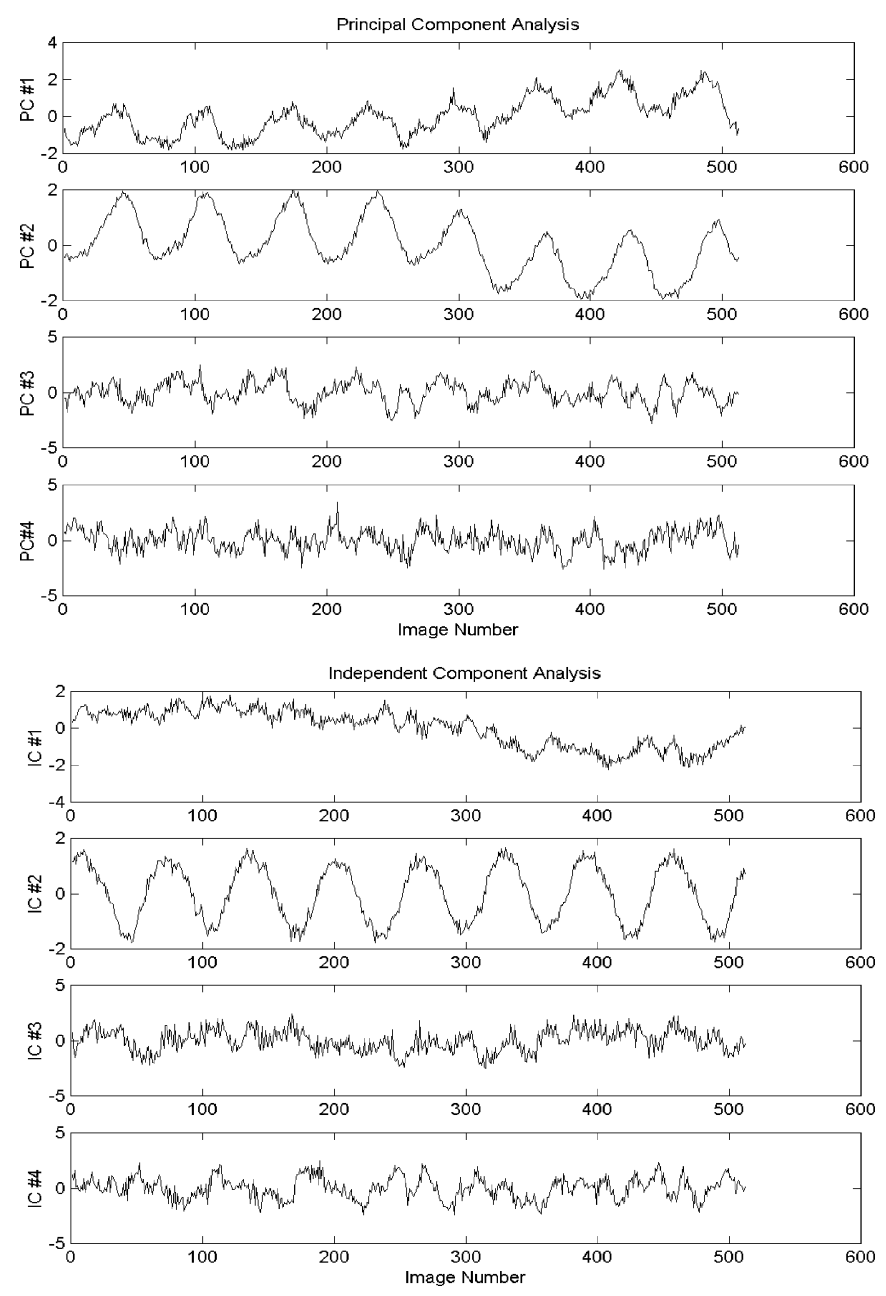

Fig. 14. PCA/ICA results after processing with the proposed method. The improvement from the previous figure is evident particularly in the ICA results.
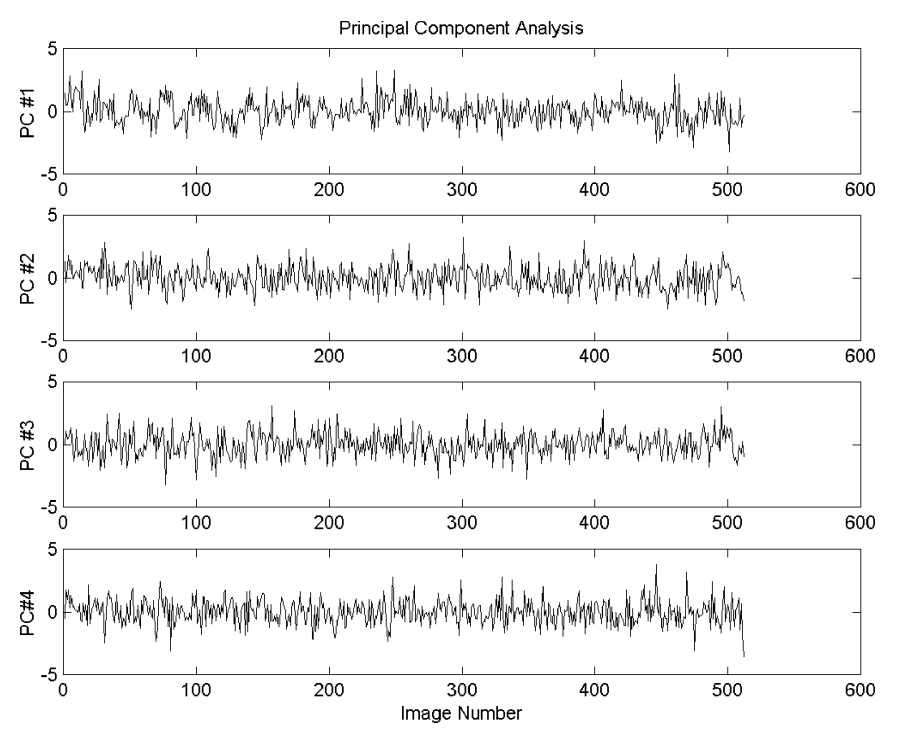

Fig. 15. PCA analysis of the difference signal. No periodic component can be observed indicating the random nature of the removed signal.

computations. For efficient implementation, the noise model is estimated once and the denoising is implemented only for the regions of interest. Given that the denoising of a particular time course is performed completely independently from the others, the proposed method can readily take advantage of parallel processing when available.

In developing the proposed technique, very few assumptions about the nature of the noise model and no assumptions about the deterministic signal components are made. This is an obvious advantage compared to previous techniques that rely on signal models to construct the denoising filter (cf. [4]). Given that the noise model parameters can be adaptively estimated from background areas within the fMRI data set, the implementation of the new technique is expected to maintain robustness.

The new technique provides a statistical control over noise removal using a single parameter $\alpha$. This allows the user to customize its use to the specific data analysis tool of his/her choice. At the same time, it allows the comparison of implementations between different groups using this unambiguous reference.

The results indicate that new technique enables robust use of PCA and ICA. The main advantages of using the new technique come as a direct result of the conditioning of the input signals to match the requirements of these analysis methods. That is, to make sure that the number of independent sources is less than the number of signals. This is reflected on the results as better convergence characteristics as well as less mixing between remaining noise components and the deterministic components in the output of such analyses. The results emphasize the non-Gaussian nature of the deterministic fMRI signal components. This is evident from comparing the outputs from ICA as compared to those from PCA. This suggests that a study of this characteristic of the signal components should be addressed in a future study using the new technique as a preprocessing step. A similar study can be performed using the replicator dynamics approach, which bears similarity in its assumptions and limitations to the PCA and ICA [22].

The limitations of the new technique include two parts, namely, the noise in phase component, and the effect of the clipping in the subtracted power spectrum. The first is partially removed when only the real part of the signal is used as the denoised signal, which effectively means that the phase values in the negative and positive frequency are averaged. On the other hand, the effect of spectral clipping can be modeled as an addition of another random signal that accounts for the clipped parts. This results in a degradation of the results that was deemed insignificant by our experiments. Further investigation of these limitations is required to improve on the results of the proposed technique.

\section{CONCLUSION}

A new signal denoising technique was proposed for fMRI signals. The new strategy based on spectral subtraction method is adaptive and simple to implement while offering a substantial improvement of the SNR. The implementation was described and its performance was demonstrated using computer simulations and real data. The use of the proposed technique as a preprocessing step was also shown to substantially improve the performance of PCA and ICA in analyzing fMRI data sets. Further work is needed to investigate the potential of the new technique in different clinical applications. 


\section{APPENDIX \\ RELATIONSHIP BETWEEN SPECTRAL SUbTRACTION AND THE OPTIMUM WIENER FILTER}

The spectral subtraction technique described in this paper can be expressed in the form

$$
\hat{P}_{D D}=\hat{P}_{S S} \cdot\left(\frac{\hat{P}_{S S}-P_{n n}}{P_{n n}}\right)
$$

where $\hat{P}_{S S}=\left\{|S(\omega)|^{2}\right\}$ is the instantaneous (single periodogram) estimate power spectrum of the original signal, $P_{n n}=E\left\{|N(\omega)|^{2}\right\}$ is the ensemble-averaged (true) estimate of the power spectrum of noise, and the result is $\hat{P}_{D D}=\left\{\left|S_{D}(\omega)\right|^{2}\right\}$, the instantaneous estimate of the power spectrum of the original signal. The term in parentheses can be considered as a zero-phase "filter" that acts as a SNR-dependent attenuator whereby the numerator is considered as an estimate of the true signal power spectrum while the denumerator is the true noise power spectrum. The problem of filtering a deterministic signal from additive noise has an optimum solution furnished by the Wiener filter theory given as

$$
\hat{P}_{D D}=\hat{P}_{S S} \cdot\left(\frac{P_{S S}-P_{n n}}{P_{n n}}\right) .
$$

Here, $P_{S S}=E\left\{|S(\omega)|^{2}\right\}$ is the true power spectrum of the original signal. Hence, we observe that the spectral subtraction is an approximation of the optimum Wiener filter in which the true power spectrum of the signal is replaced by the instantaneous estimate of the original signal power spectrum [10]. The result of this approximation is that the output will be identical in the mean to the true signal but with variance determined by the variance of this instantaneous estimate [20]. That is, the proposed methodology is an unbiased estimator of the true signal provided by the optimum Wiener filter [19].

\section{ACKNOWLEDGMENT}

The author would like to thank Dr. S. Ngan, Dr. S. Laconte, Dr. X. Hu, and Dr. T. Parrish for sharing their data and results and for helpful discussions.

\section{REFERENCES}

[1] W. W. Orrison, J. D. Lewine, J. A. Sanders, and M. F. Hartshorne, Functional Brain Imaging. St. Louis, MO: Mosby-Year Book, 1995.

[2] S. Ogawa, D. W. Tank, R. Menon, J. M. Ellermann, S.-G. Kim, H. Merkle, and K. Ugurbil, "Intrinsic signal changes accompanying sensory stimulation: functional brain mapping with magnetic resonance imaging," Proc. Nat. Acad. Sci. USA, vol. 89, pp. 5951-5955, 1992.

[3] R. L. Buckner, P. A. Bandettini, K. M. O'Craven, R. L. Savoy, S. E. Petersen, M. E. Raichle, and B. R. Rosen, "Detection of cortical activation during averaged single trials of a cognitive task using functional magnetic resonance imaging," Proc. Nat. Acad. Sci. USA, vol. 93, pp. 14 878-14 883, 1996.

[4] S. M. LaConte, S. Ngan, and X. Hu, "Wavelet transform-based Wiener filtering of event-related fMRI data," Magn. Reson. Med., vol. 44, pp. 746-757, 2000.

[5] T. H. Le and X. Hu, "Retrospective estimation and correction of physiological artifacts in fMRI by direct extraction of physiological activity from MR data," Magn. Reson. Med., vol. 35, pp. 290-298, 1996.

[6] B. A. Ardekani, J. Kershaw, K. Kashikura, and I. Kanno, "Activation detection in functional MRI using subspace modeling and maximum likelihood estimation," IEEE Trans. Med. Imag., vol. 18, pp. 101-114, Feb. 1999.
[7] M. Svensen, F. Kruggel, and Y. von Cramon, "Probabilistic modeling of single-trial fMRI data," IEEE Trans. Med. Imag., vol. 19, pp. 25-35, Jan. 2000.

[8] C. Goutte, F. A. Nielsen, and L. K. Hansen, "Modeling the haemodynamic response in fMRI using smooth FIR filters," IEEE Trans. Med. Imag., vol. 19, pp. 1188-1201, Feb. 2000.

[9] A. F. Sole, S. Ngan, G. Sapiro, X. Hu, and A. Lopez, "Anisotropic 2-D and 3-D averaging of fMRI signals," IEEE Trans. Med. Imag., vol. 20, pp. 86-93, Feb. 2001.

[10] S. V. Vaseghi, Advanced Signal Processing and Digital Noise Reduction. New York: Wiley, 1996.

[11] A. M. Law and W. D. Kelton, Simulation, Modeling \& Analysis, 2nd ed. New York: McGraw-Hill, 1991.

[12] A. Hyvarinen, J. Karhunen, and E. Oja, Independent Component Analysis. New York: Wiley, 2001.

[13] S. Makeig, T. Jung, D. Ghahremani, and T. J. Sejnowski, "Independent Component Analysis of Simulated ERP Data," Inst. Neural Computation, Tech. Rep. INC-9606.

[14] S. Makeig, M. Westerfield, T. Jung, J. Covington, J. Townsend, T. J. Sejnowski, and E. Courchesne, "Functionally independent components of the late positive event-related potential during spatial attention," $J$. Neurosci., vol. 19, no. 7, pp. 2665-2680, 1999.

[15] M. J. Mckeown, T. Jung, S. Makeig, G. Brown, S. S. Kindermann, T. Lee, and T. J. Sejnowski, "Spatially independent activity patterns in functional MRI data during the Stroop color-naming task," Proc. Nat. Acad. Sci., USA, vol. 95, pp. 803-810, 1998.

[16] M. J. McKeown, S. Makeig, G. G. Brown, T. Jung, S. S. Kindermann, A. J. Bell, and T. J. Sejnowski, "Analysis of fMRI data by blind separation into independent spatial components," Hum. Brain Map., vol. 6, pp. 160-188, 1998.

[17] V. D. Calhoun, T. Adali, G. D. Pearlson, P. C. M. van Zijl, and J. J. Pekar, "Independent component analysis of fMRI data in the complex domain," Magn. Reson. Med., vol. 48, pp. 180-192, 2002.

[18] A. Hyvarinen, "Fast and robust fixed-point algorithms for independent component analysis," IEEE Trans. Neural Networks, vol. 10, pp. 626-634, May 1999.

[19] B. P. Lathi, Modern Digital and Analog Communication Systems, 2nd ed. Oxford, U.K.: Oxford Univ. Press, 1989.

[20] C. S. Burrus, J. H. McClellan, A. V. Oppenheim, T. W. Parks, R. W. Schafer, and H. W. Schuessler, Computer-Based Excercises for Signal Processing Using Matlab. Englewood Cliffs, NJ: Prentice Hall, 1994

[21] L. Freire, A. Roche, and J.-F. Mangin, "What is the best similarity measure for motion correction in fMRI time series?," IEEE Trans. Med. Imag., vol. 21, May 2002.

[22] G. Lohmann and S. Bohn, "Using replicator dynamics for analyzing fMRI data of the human brain," IEEE Trans. Med. Imag., vol. 21, May 2002.

[23] H. Gudbjartsson and S. Patz, "The Rician distribution of noisy MRI data," Magn. Reson. Med., vol. 34, pp. 910-914, 1995.

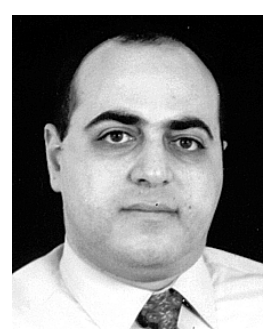

Yasser M. Kadah (M'01-SM'03) was born in Giza, Egypt in 1967. He received the B.Sc. and M.Sc. degrees from the Biomedical Engineering Department, Cairo University, in 1989 and 1992 respectively. He received the Ph.D. degree in biomedical engineering from the University of Minnesota, Twin Cities, in 1997.

He worked as a Research Assistant with the Department of Radiology, University of Minnesota Medical School in 1996-1997 and as a Postdoctoral Fellow at the Center of Magnetic Resonance Research at the University of Minnesota in 1998. He has been with the Biomedical Engineering department at Cairo University since 1998 where he is currently an Associate Professor. He worked between 1998 and 2002 as the Director of Research and Development at IBE Technologies, Giza, Egypt. He is on leave of absence since December 2002 to work at the Biomedical Imaging Technology Center of the W. H. Coulter department of Biomedical Engineering at Emory University and Georgia Institute of Technology. His research interests include medical imaging, in particular, MRI and ultrasound, and multidimensional signal processing for biomedical applications.

Dr. Kadah has received several awards and recognitions including the record for highest undergraduate GPA in the department of Biomedical Engineering at Cairo University and the IDB Merit Scholarship (1993-1996) as well as the E. K. Zaviosky Stipend from the International Society of Magnetic Resonance in Imaging (2002). His biography was selected to appear in Marquis Who's Who in the World (2002), He is an active member of the ISMRM and SPIE. 